\title{
Effective Treatment of a Melanoma Patient with Hemophagocytic Lymphohistiocytosis after Nivolumab and Ipilimumab Combined Immunotherapy
}

\author{
Renata Pacholczak-Madej ${ }^{1,2}$, Aleksandra Grela-Wojewoda ${ }^{1}$, \\ Joanna Lompart ${ }^{1}$, Beata Żuchowska-Vogelgesang ${ }^{1}$, Marek Ziobro' \\ ${ }^{1}$ Department of Clinical Oncology, The Maria Sklodowska-Curie National \\ Research Institute of Oncology, Cracow, Poland; \\ ${ }^{2}$ Department of Anatomy, Jagiellonian University Medical College, Cracow, Poland \\ Received August 23, 2021; Accepted January 31, 2022.
}

Key words: Melanoma - Immune-related adverse events - Hemophagocytic lymphohistiocytosis - Nivolumab and ipilimumab

\begin{abstract}
Immune checkpoint inhibitors have significantly improved the prognosis of melanoma patients. However, these therapies may trigger unexpected immunerelated adverse events (irAEs), which are challenging in making the proper diagnosis and providing treatment. Hematological toxicities are possible irAEs, but were poorly evaluated in clinical trials and treatment recommendations of this specific complications are limited. We present a stage IV melanoma patient who developed an extremely rare toxicity - hemophagocytic lymphohistiocytosis (HLH) after the $4^{\text {th }}$ course of combined immunotherapy with nivolumab and ipilimumab. The patient was steroid resistant and only the treatment with various immunosuppressive agents provided control of the disease and finally melanoma regression. In this report, we evaluated the methods of HLH treatment and described our modification of available protocols. Immediate immunosuppression can be life-saving and due to rarity of this condition as well as lack of specific recommendations, every report is valuable for clinicians, especially when treatment was effective.
\end{abstract}

Mailing Address: Marek Ziobro, MD., PhD., Garncarska 11 Street, 31-115 Cracow, Poland; e-mail: mz5@wp.pl 


\section{Introduction}

Immune checkpoint inhibitors ( $\mathrm{ICls}$ ) have changed the treatment landscape of melanoma patients. However, these therapies may trigger unexpected immunerelated adverse events (irAEs) with various clinical presentations. The frequency of grade 3/4 (G3/G4) irAEs according to Common Terminology Criteria for Adverse Events (CTCAE) in KEYNOTE-006 occurred in 17\% of the patients treated with pembrolizumab, in CheckMate- 067 affected $59 \%$ and $23 \%$ of the patients in nivolumab plus ipilimumab, and nivolumab groups, respectively (Robert et al., 2015; Larkin et al., 2019).

Hematological toxicities are possible irAEs, but were poorly evaluated in clinical trials. In the systematic review the overall incidence of all-grade anemia, neutropenia and thrombocytopenia during anti-programmed cell death (ligand) protein 1 - anti-PD-(L) 1 - inhibitors were $9.8 \%, 0.94 \%$, and $2.8 \%$, respectively. Febrile neutropenia occurred in $0.45 \%$ patients (Petrelli et al., 2018). In the analysis of World Health Organization's pharmacovigilance database of individual-casesafety reports (VigiBase), various types of hematologic conditions associated with anti-cytotoxic T lymphocyte antigen-4 (CTLA-4; ipilimumab, tremelimumab), antiPD-1 (nivolumab, pembrolizumab), and anti-PD-L1 (avelumab, durvalumab, and atezolizumab) treatment were reported, such as: hemolytic anemia $(n=68)$, immune thrombocytopenic purpura $(n=57)$, hemophagocytic lymphohistiocytosis $(\mathrm{HLH}$, $n=26)$, aplastic anemia $(n=10)$, and pure red cell aplasia $(n=7)$ (Davis et al., 2019). $\mathrm{HLH}$ was most commonly associated with melanoma $(n=15,58 \%)$ during treatment with anti-CTLA-4 therapy ( $n=13,50 \%)$, occurred earlier (median 26 days), and had a significantly higher rate of fatalities $(n=6,23 \%)$ than other hematologic toxicities. Unfortunately, there is no standard treatment for hematologic irAEs which might be potentially fatal, and there were only 2 cases related to anti-CTLA-4 plus anti-PD-1 therapy reported in VigiBase (Davis et al., 2019).

In this case report, we present a patient with advanced melanoma, whose diagnosis of specific irAEs was challenging for our team. The patient developed HLH after the $4^{\text {th }}$ course of combined immunotherapy with nivolumab and ipilimumab and was primary resistant to steroids. She required treatment with various immunosuppressive drugs, but luckily, the treatment provided symptom resolution and remission of melanoma. We believe that due to rarity of this condition and lack of specific recommendations, every report is valuable for clinicians, especially when treatment was effective.

\section{Case report}

We report a case of 57-year-old woman with a stage IV B-Raf proto-oncogene (BRAF)-wild-type melanoma with an inoperable tumour in the left groin area (Figure 1A). Due to a good performance status (PS 1) and lack of severe comorbidities, she started the combined immunotherapy with nivolumab $(1 \mathrm{mg} / \mathrm{kg})$ and ipilimumab $(3 \mathrm{mg} / \mathrm{kg})$. However, after the fourth cycle, the patient's general 

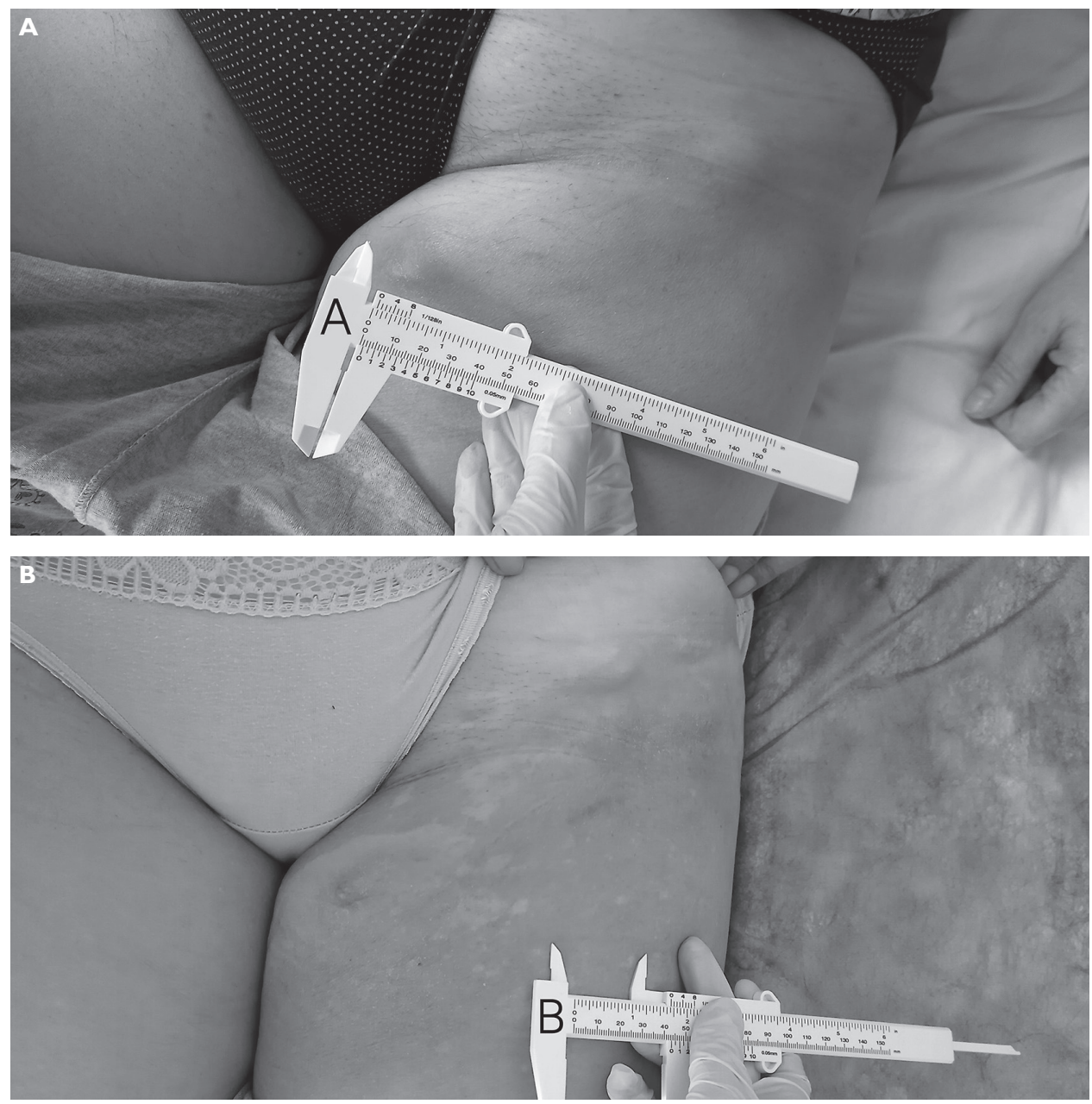

Figure 1 - A) Tumour in the left groin area before the beginning of the treatment, and B) after 4 courses of combined immunotherapy with signs of vitiligo (scale in centimeters).

condition started to deteriorate with persistent, high-spiking fever $>40.0^{\circ} \mathrm{C}$, general malaise, dyspnea at rest and elevated markers of inflammation and lactate dehydrogenase (LDH). Having a suspicion of irAEs, we started the initial treatment with prednisone $1 \mathrm{mg} / \mathrm{kg}$ without any improvement. Hence, the patient was admitted to our department for further work-up. Because of the ongoing SARS-CoV-2 pandemic, we excluded coronavirus infection by polymerase chain reaction (PCR) test. Remarkable laboratory findings included elevated markers of inflammation (C-reactive protein $168 \mathrm{ng} / \mathrm{ml}$, procalcitonin $0.36 \mathrm{ng} / \mathrm{ml}$ ) with negative blood cultures, transaminase G3 according to CTCAE, active urinary 


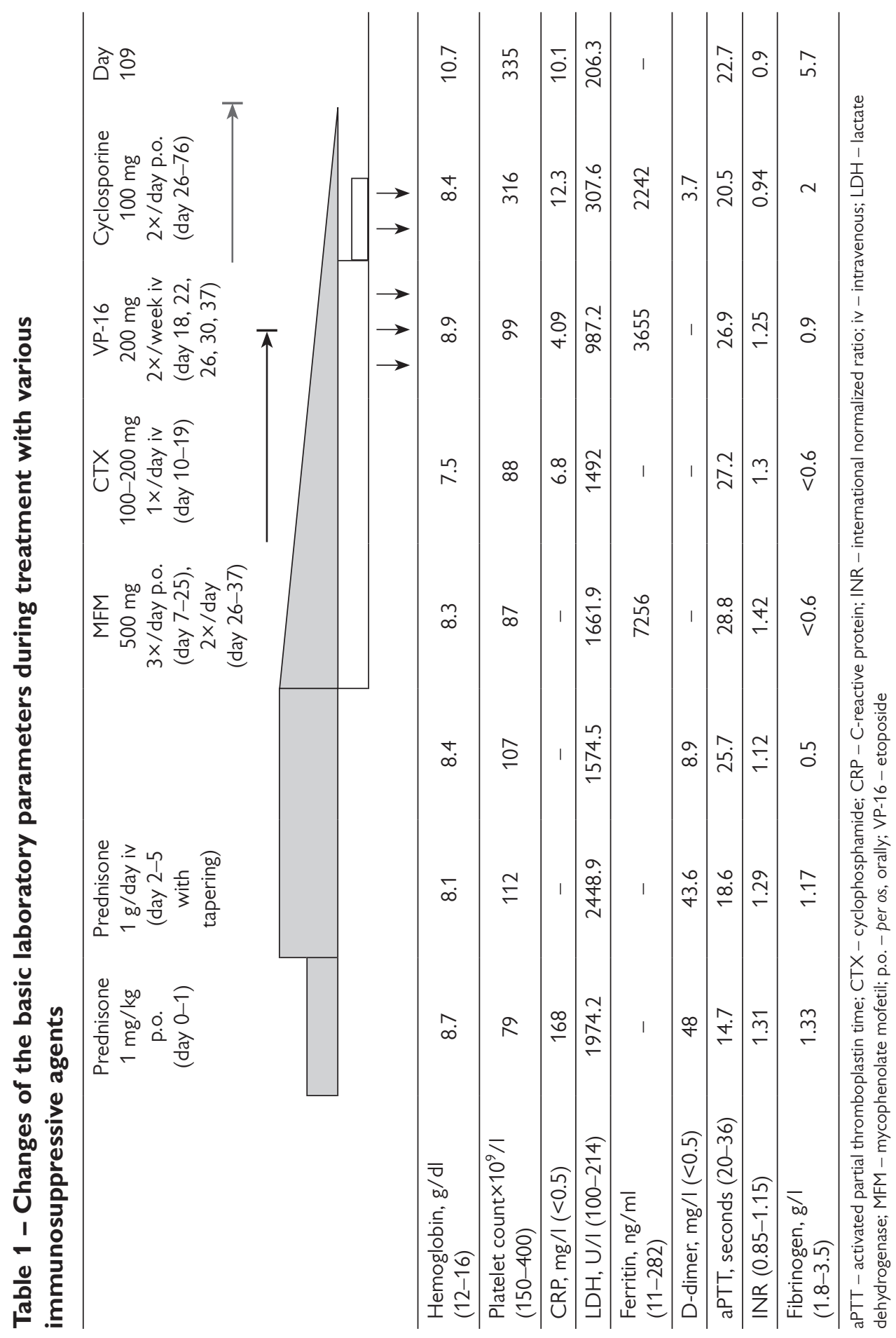

Pacholczak-Madej R.; Grela-Wojewoda A.; Lompart J.; Żuchowska-Vogelgesang B.; Ziobro M. 
sediment, elevated lactate dehydrogenase 2,448.0 U/I, with features of disseminated intravascular coagulation (DIC) - D-dimers $43.6 \mathrm{ng} / \mathrm{l}$, thrombocytes $79-112 \times 10^{9} / \mathrm{l}$, hemoglobin $8.1 \mathrm{~g} / \mathrm{dl}$, fibrinogen $1.17 \mathrm{~g} / \mathrm{l}$, activated partial thromboplastin time (aPTT) 18.6 seconds, international normalized ratio (INR) 1.29 (reference values are presented in Table 1). In response, we started pulses of methylprednisolone $1,000 \mathrm{mg}$ during 5 days observing amelioration of the patient's condition and symptom remission, but without any major improvement in laboratory parameters with ongoing activation of coagulation (for detailed parameters see Table 1). The patient required a transfusion of fresh frozen plasma in order to prevent her from purpura. Computed tomography (CT) revealed the pleural effusion on the left side and splenomegaly without any evident signs of progression of the disease. We gradually tapered the dose of steroids and introduced mycophenolate mofetil $500 \mathrm{mg} 3$ times per day and additionally cyclophosphamide 100-200 mg for 9 days with stabilization of platelets, but still an extremely low fibrinogen level. After 15 days of immunosuppressive treatment, we determined the ferritin level which was significantly increased $(7,256 \mathrm{ng} / \mathrm{ml})$ together with hypertriglyceridemia of $2.85 \mathrm{mmol} / \mathrm{I}$ (reference value $<1.7 \mathrm{mmol} / \mathrm{l}$ ), splenomegaly in CT scan (12 centimeters). Hence, the patient met 5 out of 8 diagnostic criteria of HLH according to HLH-2004 protocol (Henter et al., 2007) (+ fever, cytopenias in two lines: hemoglobin and platelets) with $98.7 \%$ probability of having $\mathrm{HLH}$ as estimated using the HLH-probability calculator (HScore, available at: http://saintantoine.aphp .fr/score/) (Fardet et al., 2014). We did not perform a bone marrow biopsy due to the high probability of HLH based on abovementioned clinical and laboratory features and unstable coagulation tests. Once the diagnosis was established we started treatment according to the pediatric protocol adding etoposide $200 \mathrm{mg}$ two times per week with our modification (Table 1). After several days of the intensive immunosuppressive treatment, we observed an improvement in laboratory parameters and the patient's full recovery, thereby the patient was discharged after 20 days of hospitalization.

During outpatient treatment, the patient continued the maintenance therapy with reduced dose of mycophenolate mofetil (500 mg two times per day), cyclosporine (100 mg two times per day), low dose of steroids (prednisone $40 \mathrm{mg}$ per day with gradual tapering). We discontinued etoposide after 6 cycles due to neutropenia G2 (CTCAE). As a consequence of life-threatening toxicity G4 (CTACE), the immunotherapy was definitely stopped and the patient remained in observation. She was regularly monitored in our outpatient clinic with clinical features of tumour shrinkage in the left groin area with signs of vitiligo on the left thigh (Figure 1B) and systematic improvement in basic laboratory parameters (Table 1). Immunosuppressive agents were gradually discontinued and finally stopped after 10 weeks since the HLH diagnosis. Additionally, the patient was referred to radiotherapy of the remaining tumour in the left inguinal region. She received 50 Gray (Gy) in 20 fractions using volumetric modulated arc therapy (VMAT). 
CT scan performed 3 months after discontinuation of immunotherapy revealed a partial remission of the tumour. The patient remains in a good clinical condition and remission of melanoma.

\section{Discussion}

$\mathrm{HLH}$ is a disease on the spectrum of cytokine storm syndrome with uncontrolled inflammation, which might be fatal in case of an incorrect treatment. We may distinguish two types: primary HLH - in which a genetic defect causes a lack of cytotoxic activity of T lymphocytes and natural killer cells, and acquired - associated with infections, malignancies, and autoinflammatory disorders (Machowicz and Basak, 2020). Nowadays, an additional triggering factor is the treatment with $\mathrm{ICls}$, when we purposely release the blockade of the immune system.

The name HLH comes from hemophagocytosis, which is the phagocytosis of blood cells by activated macrophages observed in the bone marrow smear or in cytology from the other, involved organ. However, confirmation of its presence is neither required nor sufficient for the diagnosis of $\mathrm{HLH}$, because not all patients have hemophagocytosis at disease onset (Gupta et al., 2008). Due to the rapid onset and often fulminant progress, diagnosis should not be delayed by looking for this single feature. Jordan et al. (2011) considered hemophagocytosis as the less important diagnostic criteria. Hence, we did not perform the bone marrow biopsy in the presented case. The diagnosis of HLH is made on the basis of clinical and laboratory features (HLH-2004 diagnostic criteria), such as: fever, splenomegaly, cytopenia in $\geq 2$ lines (neutrophils $<1.0 \times 10^{9} /$ /; hemoglobin $<9 \mathrm{~g} / \mathrm{dl}$; platelet count $<100 \times 10^{9} /$ I), hypofibrinogenemia $(\leq 1.5 \mathrm{~g} / \mathrm{l}[150 \mathrm{mg} / \mathrm{dl}])$ and/or hypertriglyceridemia $(\geq 3 \mathrm{mmol} / \mathrm{I}$ $[265 \mathrm{mg} / \mathrm{dl}]$ ), hemophagocytosis, ferritin $\geq 500 \mathrm{ng} / \mathrm{ml}$, low NK-cell activity, soluble cluster of differentiation 25 (sCD25/soluble interleukin 2 receptor) $\geq 2,400 \mathrm{U} / \mathrm{ml}$ with fulfilment of at least 5 out of 8 (La Rosée et al., 2019). Soluble CD25 is a useful marker of inflammation, but is not available at our institution, similarly to NK-cell activity. However, even without these parameters, fulfilment of the remaining criteria was sufficient to establish the proper diagnosis.

The treatment of HLH depends on the underlying trigger and severity of symptoms. General recommendation states that in case of rapid deterioration of the patient's methylprednisolone pulses or dexamethasone $10 \mathrm{mg} / \mathrm{m}^{2}$ should be initiated with or without intravenous immunoglobulins (1.6 g/ kg split over 2-3 days) (Machowicz and Basak, 2020). This approach may be sufficient in moderate HLH. In more severe cases the standards of treatment are protocols adopted from the pediatric population - HLH-94 and HLH-2004 (Henter et al., 2007; La Rosée et al., 2019). Both of these regimens are based on etoposide $\left(2 \times 150 \mathrm{mg} / \mathrm{m}^{2} /\right.$ week $1-2$, and $1 \times 150 \mathrm{mg} / \mathrm{m}^{2} /$ week 3-8), dexamethasone $\left(10 \mathrm{mg} / \mathrm{m}^{2}\right.$ with tapering dose) and cyclosporine (aiming at blood levels of around $200 \mu \mathrm{g} / \mathrm{l}$ ). The main difference is the initiation of cyclosporine - concomitantly from the beginning of the treatment (in HLH-2004) or as a maintenance after 8 weeks (in HLH-94) (Henter et al., 2007; 
La Rosée et al., 2019). Due to the toxicity of HLH-94 protocol in adults, Henter et al. (2006) proposed a reduction of the etoposide dose to $50-100 \mathrm{mg} / \mathrm{m}^{2}$ administered once per week.

Unfortunately, there is a lack of specific recommendations for ICls-related HLH. European Society of Medical Oncology recommends the initiation of high-dose corticosteroids and other immunosuppressive drugs with close collaboration with a hematologist (Haanen et al., 2017). In the literature there are available case reports in which treatment interruption or corticosteroids alone were used with sufficient response (Sadaat and Jang, 2018; Mizuta et al., 2020; Gambichler et al., 2021). In melanoma patients HLH can be also attributed to a solid tumour as a first presentation of the disease (Stabler et al., 2017), Epstein-Barr virus (EBV) infection (Davis et al., 2019) and can be associated with BRAF/MEK inhibitors (Samaran et al., 2020; Dudda et al., 2021). These cases represented rather moderate HLH where treatment discontinuation, basic supportive care (intravenous rehydration and paracetamol), and low dose steroids (or even without steroids prescription) (Samaran et al., 2020) were sufficient methods of treatment. In 19\% of cases in VigiBase, concomitant Epstein-Barr virus infection was reported, suggesting a potentially augmented immune response in case of chronic underlying infection (Davis et al., 2019). Paradoxically, nivolumab has been also used in the treatment of relapsed/refractory EBV-associated HLH (Liu et al., 2020). In the presented case, the patient was steroid-refractory and only the treatment with $\mathrm{HLH}-2004$ protocol with our modification - an additional short course of cyclophosphamide with cyclosporine and mycophenolate as maintenance, provided control of the disease.

\section{Conclusion}

Immune-related adverse events can be challenging in making diagnosis. Although $\mathrm{HLH}$ is potentially fatal disease, it can be recognized by some easily accessible laboratory parameters. Diagnostic procedures should not delay immediate administration of high dose steroids, which in many cases is a sufficient treatment. However, some patients can be steroid-refractory and require additional treatment with immunosuppressive agents. Proper immunosuppression can be life-saving and in spite of the immunotherapy withdrawal may provide melanoma regression as in the presented patient.

\section{References}

Davis, E. J., Salem, J., Young, A., Green, J. R., Ferrell, P. B., Ancell, K. K., Johnson, D. B. (2019) Hematologic complications of immune checkpoint inhibitors. Oncologist 24(5), 584-588.

Dudda, M., Mann, C., Heinz, J., Schmidgen, I., Weid, F., Kühn, M., Loquai, C. (2021) Hemophagocytic lymphohistiocytosis of a melanoma patient under BRAF/MEK-inhibitor therapy following anti-PD1 inhibitor treatment: A case report and review to the literature. Melanoma Res. 31(1), 81-84.

Fardet, L., Galicier, L., Lambotte, O., Marzac, C., Aumont, C., Chahwan, D., Hejblum, G. (2014) Development and validation of the HScore, a score for the diagnosis of reactive hemophagocytic syndrome. Arthritis Rheumatol. 66(9), 2613-2620. 
Gambichler, T., Rached, N. A., Nowack, N., Behle, B., Susok, L. (2021) Hemophagocytic lymphohistiocytosis after initiation of combined immunotherapy for metastatic melanoma. Ann. Hematol. Oncol. 8(4), 1337.

Gupta, A., Weitzman, S., Abdelhaleem, M. (2008) The role of hemophagocytosis in bone marrow aspirates in the diagnosis of hemophagocytic lymphohistiocytosis. Pediatr. Blood Cancer 50(2), 192-194.

Haanen, J. B. A. G., Carbonnel, F., Robert, C., Kerr, K. M., Peters, S., Larkin, J., Jordan, K. (2017) Management of toxicities from immunotherapy: ESMO Clinical Practice Guidelines for diagnosis, treatment and followup. Ann. Oncol. 28, iv119-iv142 (Suppl. 4).

Henter, J. I., Horne, A., Aricó, M., Egeler, R. M., Filipovich, A. H., Imashuku, S., Ladisch, S., McClain, K., Webb, D., Winiarski, J., Janka, G. (2007) HLH-2004: Diagnostic and therapeutic guidelines for hemophagocytic lymphohistiocytosis. Pediatr. Blood Cancer 48(2), 124-131.

Henter, J. I., Chow, C. B., Leung, C. W., Lau, Y. L. (2006) Cytotoxic therapy for severe avian influenza A (H5N1) infection. Lancet 367(9513), 870-873.

Jordan, M. B., Allen, C. E., Weitzman, S., Filipovich, A. H., McClain, K. L. (2011) How I treat hemophagocytic lymphohistiocytosis. Blood 118(15), 4041-4052.

La Rosée, P., Horne, A., Hines, M., von Bahr Greenwood, T., Machowicz, R., Berliner, N., Birndt, S., GilHerrera, J., Girschikofsky, M., Jordan, M. B., Kumar, A., van Laar, J. A. M., Lachmann, G., Nichols, K. E., Ramanan, A. V., Wang, Y., Wang, Z., Janka, G., Henter, J. I. (2019) Recommendations for the management of hemophagocytic lymphohistiocytosis in adults. Blood 133(23), 2465-2477.

Larkin, J., Chiarion-Sileni, V., Gonzalez, R., Grob, J.-J., Rutkowski, P., Lao, C. D., Wolchok, J. D. (2019) Five-year survival with combined nivolumab and ipilimumab in advanced melanoma. N. Engl. J. Med. 381(16), 1535-1546.

Liu, P., Pan, X., Chen, C., Niu, T., Shuai, X., Wang, J., Liu, T. (2020) Nivolumab treatment of relapsed/ refractory Epstein-Barr virus-associated hemophagocytic lymphohistiocytosis in adults. Blood 135(11), 826-833.

Machowicz, R., Basak, G. (2020) How can an internal medicine specialist save a patient with hemophagocytic lymphohistiocytosis (HLH)? Pol. Arch. Intern. Med. 130(5), 431-437.

Mizuta, H., Nakano, E., Takahashi, A., Koyama, T., Namikawa, K., Yamazaki, N. (2020) Hemophagocytic lymphohistiocytosis with advanced malignant melanoma accompanied by ipilimumab and nivolumab: A case report and literature review. Dermatol. Ther. 33(3), e13321.

Petrelli, F., Ardito, R., Borgonovo, K., Lonati, V., Cabiddu, M., Ghilardi, M., Barni, S. (2018) Haematological toxicities with immunotherapy in patients with cancer: A systematic review and meta-analysis. Eur. J. Cancer 103, 7-16.

Robert, C., Schachter, J., Long, G. V., Arance, A., Grob, J. J., Mortier, L., Ribas, A. (2015) Pembrolizumab versus ipilimumab in advanced melanoma. N. Engl. J. Med. 372(26), 2521-2532.

Sadaat, M., Jang, S. (2018) Hemophagocytic lymphohistiocytosis with immunotherapy: Brief review and case report. J. Immunother. Cancer 6(1), 49.

Samaran, Q., Belakebi, D., Theret, S., Becquart, O., Girard, C., Du Thanh, A., Guillot, B., Lesage, C., Dereure, O. (2020) Hemophagocytic lymphohistiocytosis in advanced melanoma treated with dabrafenib and trametinib combination: two cases. Melanoma Res. 30(5), 519-523.

Stabler, S., Becquart, C., Dumezy, F., Terriou, L., Mortier, L. (2017) Hemophagocytic lymphohistiocytosis in patients with metastatic malignant melanoma. Melanoma Res. 27(4), 377-379.

Pacholczak-Madej R.; Grela-Wojewoda A.; Lompart J.; Żuchowska-Vogelgesang B.; Ziobro M. 